\title{
Integrative genomic analysis identifies epigenetic marks that mediate genetic risk for epithelial ovarian cancer
}

Devin C Koestler ${ }^{1}$, Prabhakar Chalise ${ }^{1}$, Mine S Cicek ${ }^{3}$, Julie M Cunningham ${ }^{4}$, Sebastian Armasu ${ }^{3}$, Melissa C Larson ${ }^{3}$, Jeremy Chien ${ }^{2}$, Matthew Block ${ }^{5}$, Kimberly R Kalli ${ }^{5}$, Thomas A Sellers ${ }^{6}$, Brooke L Fridley ${ }^{1 *}$ and Ellen L Goode ${ }^{3}$

\begin{abstract}
Background: Both genetic and epigenetic factors influence the development and progression of epithelial ovarian cancer (EOC). However, there is an incomplete understanding of the interrelationship between these factors and the extent to which they interact to impact disease risk. In the present study, we aimed to gain insight into this relationship by identifying DNA methylation marks that are candidate mediators of ovarian cancer genetic risk.

Methods: We used 214 cases and 214 age-matched controls from the Mayo Clinic Ovarian Cancer Study. Pretreatment, blood-derived DNA was profiled for genome-wide methylation (Illumina Infinium HumanMethylation27 BeadArray) and single nucleotide polymorphisms (SNPs, Illumina Infinium HD Human610-Quad BeadArray). The Causal Inference Test (CIT) was implemented to distinguish CpG sites that mediate genetic risk, from those that are consequential or independently acted on by genotype.

Results: Controlling for the estimated distribution of immune cells and other key covariates, our initial epigenome-wide association analysis revealed 1,993 significantly differentially methylated CpGs that between cases and controls (FDR, $q<0.05)$. The relationship between methylation and case-control status for these 1,993 CpGs was found to be highly consistent with the results of previously published, independent study that consisted of peripheral blood DNA methylation signatures in 131 pretreatment cases and 274 controls. Implementation of the CIT test revealed $17 \mathrm{CpG/SNP}$ pairs, comprising 13 unique CpGs and 17 unique SNPs, which represent potential methylation-mediated relationships between genotype and EOC risk. Of these $13 \mathrm{CpGs}$, several are associated with immune related genes and genes that have been previously shown to exhibit altered expression in the context of cancer.
\end{abstract}

Conclusions: These findings provide additional insight into EOC etiology and may serve as novel biomarkers for EOC susceptibility.

Keywords: Integrative genomics, Ovarian cancer, Blood-based DNA methylation

\section{Background}

Epithelial ovarian cancer (EOC) is the fifth leading cause of cancer death among women in the United States and the most deadly among gynecologic malignancies. In 2013 it is estimated that 22,240 new cases of EOC will be diagnosed [1], making it one of the most common gynecologic malignancies. Along with the physical burdens

\footnotetext{
* Correspondence: bfridley@kumc.edu

${ }^{1}$ Department of Biostatistics, University of Kansas Medical Center, 3901

Rainbow Blvd, Kansas City, KS 66160, USA

Full list of author information is available at the end of the article
}

suffered by affected patients, the costs to the health care system are significant [2] with recent estimates suggesting that EOC accounts for upward of 5.1 billion dollars annually; rendering this disease as one of the most expensive cancers to treat [3]. The enormous physical, societal, and economic burdens associated with EOC along with the current lack of success in the early diagnosis of this disease, underscore the urgent need of studies aimed toward understanding the molecular basis of EOC susceptibility. 
Epigenetics refers to differences in phenotypic states that are not based on differences in the underlying DNA sequence, are potentially reversible, and are generally stably maintained during cell division. Epigenetic marks such as DNA methylation (DNAm) of cytosine residues in the context of $\mathrm{CpG}$ dinucleotides, have been extensively characterized in EOC tumor tissue and have been shown to differ between histological subtypes of ovarian cancer [4], associate with patient clinical outcomes including survival time [5] and progression [6], and have led to identification of inherited variants in $H N F 1 B$ (hepatocyte nuclear factor 1 homeobox B) as a subtype-specific susceptibility gene [7]. Despite the obvious relevance of investigating tumor-derived DNAm signatures for understanding EOC risk and prognosis, it also is clear that tumors do not develop as isolated phenomenon in their target tissue, but instead result from altered processes affecting neighboring cells and tissues, including the immune system. Thus, alterations DNAm profiles measured in peripheral blood may be useful not only in understanding the carcinogenic process and response to environmental insults, but may also provide critical insights in a systems biological view of tumorigenesis. Recent work has begun to translate these findings to clinically useful endpoints by examining the relationship between DNAm alterations and cancer risk [8-11], including ovarian cancer [12]. Yet, the retrospective nature of such studies and the assessment of DNAm peripheral blood leukocytes present significant challenges in the interpretation of the results; in particular, (a) the extent to which the identified methylation marks are consequential or are causal/mediators of disease risk and (b) potential for confounding due to heterogeneity in the underlying population of cells used for methylation assessment [13-15].

These challenges have served to motivate the application of novel analytical approaches for retrospective studies of DNA methylation that aim to distinguish epigenetic marks that are consequential or reflect alterations to the methylome driven by the tumor itself, from those that are causal or mediate tumor growth and development. In particular, a recent case-control study of rheumatoid arthritis, Liu et al., [16] utilized genotype data collected on the study subjects to identify methylation marks that fall along the casual pathway from genotype to disease status. As the potential for confounding due to cell heterogeneity represents a major bottleneck in the interpretation of blood-based studies of DNA methylation, the authors also applied a recently developed statistical methodology [13] for estimating the underlying distribution of cell types across each of the study samples, enabling them to control for the potential confounding effects of cell type heterogeneity. The overarching paradigm of this work is that genetically driven alterations in the pattern of DNAm of white blood cells can result in functional deficits in the normal functioning of immune system that modify disease susceptibility. Here, we speculated that these same might hold true for EOC risk; that genetically induced changes in the epigenetic landscape of white blood cells can alter susceptibility to EOC. Indeed, integrative genomics studies of other cancers, for example prostate cancer, indicate that the tumor epigenetic landscape is partly mediated by genetic differences, which may affect disease progression $[17,18]$. Additionally, Genome-wide association studies (GWAS) in the context of ovarian cancer have identified 11 common risk alleles [7,19-24], and six of these are located in homeobox gene clusters (HOXA, $H O X B$, and $H O X D)$, homeobox-related genes ( $H N F 1 B$ ), or genes expressed in early progenitor cells (BNC2, TERT) [20,25,26]; many developmental genes such as these are silenced by DNAm in differentiated cells and become aberrantly hypomethylated during tumorigenesis [26].

Given the well-established role of genetic variation and EOC risk and importance of examining DNA methylation in non target tissues, we attempted to leverage these findings along with the analytical framework applied in Lui et al. [16], with the goal of gaining better understanding the epigenetic basis of EOC susceptibility. Specifically, using blood-derived genome-wide epigenetic and genetic data collected on a total of 214 EOC cases and 214 controls enrolled in the Mayo Clinic Ovarian Cancer Study, we aimed to distinguish bloodbased DNA methylation markers that are candidates for mediating EOC genetic risk.

\section{Methods}

\section{Study population and sample preparation}

This study consisted of 428 women of European ancestry (214 pre-treatment invasive epithelial ovarian cancer cases and 214 controls one-to-one matched with EOC cases on the basis age (within 1-year)) between the ages of 27 and 91 enrolled in the Mayo Clinic Ovarian Cancer Study [20]. Genomic DNA was isolated from whole blood collected at the time of enrollment, using PureGene DNA isolation reagents (Gentra Systems, Minneapolis, MN), re-suspended in TE buffer, and stored at $-80^{\circ} \mathrm{C}$. Samples were bar-coded with a unique subject identification number to ensure accurate and reliable sample processing and storage. Research protocols were approved by the Mayo Clinic Institutional Review Boards, and all participants provided written informed consent.

\section{Genotype data}

Leukocyte-derived DNA was genotyped with the Illumina 610-quad Beadchip Array ${ }^{\mathrm{m}}$ according to manufacturer's protocol, at the Mayo Clinic Medical Genome Facility (Rochester, MN) by laboratory personnel blinded 
to case-control status. Detailed quality control (QC) procedures have been described elsewhere [20,27]. Briefly, Illumina's Genome Studio ${ }^{\mathrm{Tm}}$ software was used to perform automated genotype clustering and calling. Assays included duplicates and laboratory controls, which showed sample concordance of $99.93 \%$, genotype call rate of $99.7 \%$. SNPs were excluded with call rate $<95 \%$, MAF $<0.05$, HardyWeinberg Equilibrium (HWE) p-value $<10^{-4}$, or unresolved replicate errors, and samples were excluded with call rate $<95 \%$, ambiguous gender, or predicted less than $80 \%$ European ancestry. SNPnexus was used to annotate the genotyped variants [28-30].

\section{DNA methylation assays}

Leukocyte-derived DNA was assayed and underwent QC procedures at the Mayo Clinic Molecular Genome Facility (Rochester, MN). Samples were assayed in two batches, hereafter referred to as Batch $1(n=132 ; 66$ cases and matched control samples) and Batch 2 samples $(n=296$; 148 cases and matched control samples). For each sample, $1 \mu \mathrm{g}$ of genomic DNA was bisulfite modified (BSM) using the Zymo EZ96 DNA Methylation Kit (Zymo Research, Orange, CA) according to the manufacturer's protocol. Epigenome-wide assessment of DNA methylation was carried out using the Illumina Infinium HumanMethylation27 BeadChip, which is capable of interrogating the methylation status $>27,000 \mathrm{CpG}$ loci across the genome. This assay uses bisulfite-treated DNA and two site-specific probes for each marker, which bind to the associated methylated and unmethylated sequences. The intensity of the methylated probe relative to the total probe intensity (sum of methylated and unmethylated probe intensities) represents the fractional level of methylation for that specific site within a sample. Centre d'Etudes du Polymorphisme Humain (CEPH) DNA, placental DNA (positive control) and whole genome amplified (WGA) DNA (negative control) were also included $(n=9, n=12$ and $n=8$, respectively), as were technical replicates $(n=12)$. Briefly, fragmented DNA was hybridized to the BeadChips, which were then processed through a primer extension and an immunohistochemistry staining protocol to allow detection of a single-base extension reaction. Finally, BeadChips were coated and then imaged on an Illumina iScan. Analysis included control probes for assessing sampleindependent and sample-dependent performance.

\section{Methylation data pre-processing and quality control}

The methylation level of each CpG locus was calculated in GenomeStudio Methylation module (v.1.9.0) by comparing the ratio of fluorescent signal from the methylated allele to the sum from the fluorescent signal from both methylated and unmethylated alleles and scored as beta values, ranging from 0 (unmethylated) to 1 (methylated). We first excluded probes that had an rsid, were located on the Y chromosome, or were positioned at a single nucleotide polymorphism (SNPs) (dbSNP build 137), as SNPs at the same site have the potential to confound methylation assessment. We also removed CpG loci that had high beta values in BSM negative controls (defined as exceeding four standard deviations of the mean) and those that were detected in $<70 \%$ of samples (based on a detection p-value cut-off of 0.05). This left a total of 25,926 out 27,578 (94\%) of probes that passed QC. The intra-class correlation coefficients, computed based on beta values among CEPH replicates and for duplicate samples, were $>0.93$, indicating a high degree of reproducibility in our array. In addition, samples were excluded if $>25 \%$ of the probes for that sample had detection p-values that exceeded $1 \times 10^{-5}$. Following QC, 428 samples remained for analyses; including 132 samples (66 cases and matched control samples) and 296 samples (148 cases and matched control samples) in Batch 1 and Batch 2, respectively.

Next, we assessed possible plate/Beadchip/batch effects visually and through principal component analyses (PCA) [31,32]. DNAm values were logit-transformed (i.e., $\left.\log _{2}(\beta / 1-\beta)\right)$ as in previous studies to obtain the DNAm M-value for each CpG locus [33,34]. PCA represents a feature extraction technique where the methylation data is orthogonally transformed, such that the first principal component has the largest possible variance (accounts for maximal amount of variability in the methylation data), and each succeeding component, in turn has the next highest variance possible. PCA was applied to the methylation data for each batch separately $\left(n_{1}=138\right.$ and $n_{2}=296$, for Batch 1 and Batch 2, respectively) and also to the combined methylation data for both Batches $(n=428)$. The resulting top principal components (those explaining the maximal proportion of variability in DNAm) were then examined in terms of their association with technical aspects concerning the array (i.e., plate/BeadChip), and batch for the principal components estimated from the combined methylation data from the Batch 1 and Batch 2 samples. As batch was observed to be a major determinant of variability in the combined DNAm data (Additional files 1 and 2), we adjusted for batch-effects by applying the ComBat normalization method [35] using the R-package 'sva'. Combat is an empirical Bayes batch adjustment methodology that uses a location and scale adjustment for standardizing the mean and variability in methylation levels across batches. This methodology been shown to perform effectively and efficiently compared to competing batch/plate-adjustment methodologies $[36,37]$ and has become an established preprocessing step for array-based DNA methylation data [38-40]. Following the application of Combat, principal components were computed from the batch-adjusted data and inspected to ensure that batch effects had been 
successfully attenuated. In addition, within each batch we observed plate-effects (data not shown). To remove variability in DNAm due to plate, we fit a linear model to the logit-transformed methylation values for each CpG locus and included a fixed effect term for plate. The logit-transformed locus means were then added back onto the unstandardized residuals derived from these models, before back transforming values on the logit-scale to a 0 to 1 scale.

\section{Technical validation of the methylation array data}

As an orthogonal array validation, eight CpGs with a broad spectrum of percent methylation (range; 0.110.73 ) and variability (standard deviation; 0.11-0.15) were assessed using bisulfite pyrosequencing. Ninety-six samples were tested, including 45 cases and 45 controls, two samples each of WGA, BSM negative, and control samples (CpGenome ${ }^{\mathrm{Tm}}$ Universal Methylated DNA; Millipore Corporation, Billerica, MA). Primers (Additional file 3) were designed using the Pyrosequencing Assay Design Software. Genomic DNA (20-30 ng) was PCR-amplified using primers, one of which was biotinylated. Briefly, the incorporated biotinylated amplicon was immobilized on streptavidin-coated beads used to purify and render the denatured, single stranded and biotinylated PCR product. Single stranded DNA was purified using the pyrosequencing vacuum workstation. The single-stranded product was annealed to $0.3 \mu \mathrm{M}$ of the sequencing primer complementary to the single-stranded template and placed at $85^{\circ} \mathrm{C}$ for two minutes, then cooled to room temperature for five minutes. Pyrosequencing reactions were performed on Biotage PyroMark MD, and data were analyzed using PyroMD Software. Percent methylation was quantified as methylated $C$ to unmethylated $C$ ratio using the Pyro Q-CpG software, which provided automatic QC for each sample for completion of bisulfite conversion and estimates of non-converted DNA. The median Pearson correlation of methylation values between the array and pyrosequencing assays was 0.88 (Additional file 3), suggesting high concordance in the methylation array values and those generated from pyrosequencing.

\section{Cell mixture deconvolution analysis}

Recent work has demonstrated substantial differences in the DNAm signature across different leukocyte subtypes [13-15] and also differences in white blood cell proportions by EOC case-control status [41-43]. As such, heterogeneity in the underlying distribution of white blood cell types is likely to be a key confounder when examining the association between DNAm and EOC status. Using the plate- and batch-adjusted methylation data, we employed a statistical methodology [13] for inferring changes in the distribution of leukocytes based on peripheral blood DNAm signatures, in combination with a previously obtained external reference data set consisting of methylation signatures from purified leukocyte samples (i.e., B cells, natural killer (NK) cells, CD8+ T lymphocytes, CD4+ T lymphocytes, monocytes, and granulocytes) $[13,14]$. In this approach data obtained from a target set comprised of DNA methylation profiles from a heterogeneous mixture of cell populations is assumed to be a high-dimensional multivariate surrogate for the underlying distribution of cell types. Houseman et al. [13], proposed a cell mixture deconvolution methodology - similar to regression calibration - that involves the projection of DNA methylation profiles from the target set onto a reference data set, which consists of the DNA methylation signatures for isolated leukocyte subtypes. Under certain constraints, the cell mixture deconvolution approach can be used to approximate the underlying distribution of cell proportions within the target data via constrained projection. Application of this method to our data allowed us to estimate the expected difference in cell type proportions between ovarian cases and controls, as well as to predict the proportion of the aforementioned leukocyte subtypes for each of the study samples. In addition, these methods allowed us to quantify the proportion of total and systematic variability in peripheral blood DNAm explained by estimated immune cell composition.

Although this method has been shown to produce accurate and reliable estimates of the underlying distribution of cell type [44], we additionally investigated the consistency of our results with an independent study population. Specifically, we compared our estimates of the expected difference in cell type proportions between ovarian cases and controls with the results reported in Houseman et al. [13]; which consisted of the application of the cell mixture methodology using blood-derived methylation data from $n=131$ pretreatment EOC cases and $n=274$ controls [12].

\section{Causal inference test (CIT)}

In a manner similar to that described in Liu et al. [16], genotype $(\mathrm{G})$, methylation $(\mathrm{M})$, and phenotype $(\mathrm{Y})$ relationships were assessed using the causal inference test (CIT) [45] to classify them as "methylation mediated", "methylation consequential" or "independent". The CIT is comprised of a series of conditional correlation analyses that consider the possible directed relationships between a causal factor (genotype $(\mathrm{G})$ ), a potential mediator (methylation $(\mathrm{M})$ ) and an outcome (EOC status (Y)) (Figure 1A). In order for methylation $(\mathrm{M})$ to be classified as a mediator of genetic $(\mathrm{G})$ risk for EOC $(\mathrm{Y})$ the following conditions must be met: (1) G and Y are associated, (2) $G$ is associated with $M$ after adjustment for $Y$, (3) $M$ is associated with Y after adjusting for $G$, and (4) G is independent of $\mathrm{Y}$ after adjusting for $\mathrm{M}$ (Figure 1B). When 


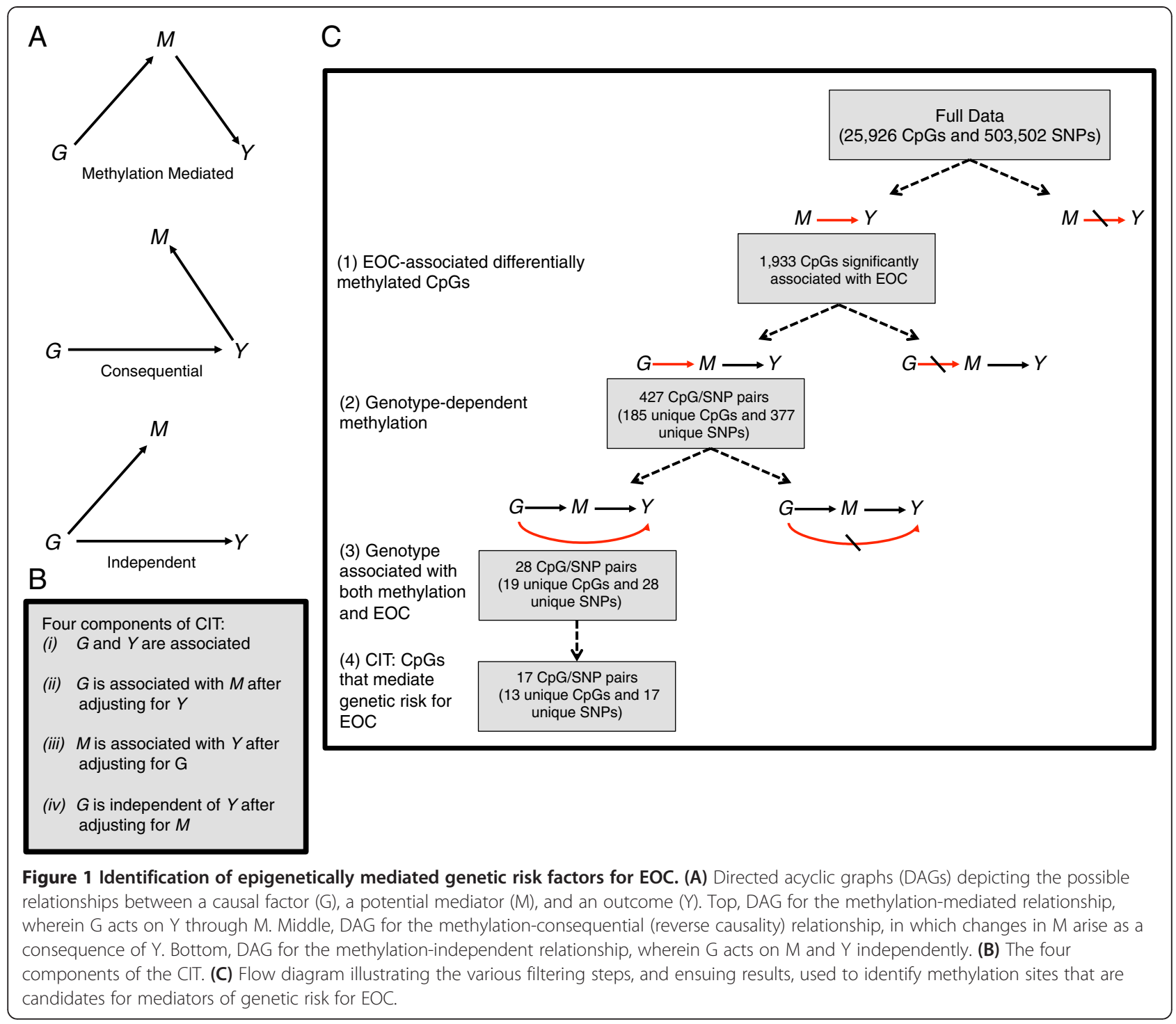

$\mathrm{M}$ is a consequence of $\mathrm{Y}$ or independently acted on by $G$ (Figure 1A), there should be no difference in the effect of $\mathrm{G}$ on $\mathrm{Y}$, when conditioning on M. However, when $\mathrm{M}$ mediates the genetic risk for EOC, conditioning on $M$ should substantially reduce the effect of G on Y $[16,45]$.

The CIT P-value was defined using the intersectionunion framework as the maximum of the component Pvalues for the first three of these conditions. G, M, and $\mathrm{Y}$ relationships were considered methylation-mediated if: the p-value obtained from the fourth condition above was $>0.05$, indicating no statistically significant association between $\mathrm{G}$ and $\mathrm{Y}$ after adjustment for $\mathrm{M}$; and the CIT P-value was $<0.05$. Where appropriate, linear and logistic regression models were used to examine the four conditions comprising the CIT.

To ease the computational burden that would ensue from examining the above conditions for every $\mathrm{G}, \mathrm{M}$, and $\mathrm{Y}$ trio $(503,502 \times 25,926 \times 4$ total tests $)$, we implemented the three step filtering procedure described in [16]. In the first step, the methylation status of each $\mathrm{CpG}$, epigenomewide, was examined for its association with Y. In step two, potential genetic regulators of methylation were identified by computing the pairwise relationship between each SNP, genome-wide, and each of $\mathrm{CpG}$ sites that were associated with $Y$ in step 1. The final step involved an examination of the relationship between $\mathrm{Y}$ and each SNP that was identified as statistically significant in step 2 . The general scheme of this analytic procedure is given in Figure 1C.

\section{Identifying ovarian cancer-associated differentially meth- ylated CpGs}

To discern differentially methylated CpGs between EOC cases and controls, we fit a series of linear regression models, which modeled the methylation M-value for each $\mathrm{CpG}$ as a function of ovarian case/control status. Models were adjusted for the estimated differential leukocyte cell 
counts described above, as well as age (continuous), current smoking status (yes vs. no), alcohol consumption (never, former, and current), study enrollment year (1999-2002, 2003, 2004, 2005, and 2006-2007), location of residence ( $\mathrm{MN}$ vs. other), parity and age at first birth (nulliparous, $1-2$ at $\leq 20 \mathrm{yrs}, 1-2$ at $>20 \mathrm{yrs}, 3+$ at $\leq 20 \mathrm{yrs}$, and $3+>20 \mathrm{yrs}$ ), and the first principal component representing within-European population sub-structure. Due to the large number of tests being performed, we corrected for multiple comparisons by computing the false discovery rate (FDR) q-value [46].

\section{Identifying genotype-dependent differentially methylated CpGs}

All epigenome-wide statistically significant (FDR q-value < 0.05 ) ovarian cancer- associated differentially methylated CpGs were subsequently examined based on their association with genotype using an additive minor-allele dosage model fit to all of the study subjects. Briefly, we used a series of linear regression models (\# ovarian cancerassociated CpGs $\times$ \# of SNPs) that modeled methylation Mvalues, as a function of the number of minor alleles for a specific SNP. Genotype-methylation associations were adjusted for multiple comparisons by computing the FDR qvalue. A less stringent FDR q-value cutoff of 0.10 was used to determine statistical significance, so as to limit false negative findings.

\section{Results}

\section{Study population}

The study population considered here consisted of 428 women of European ancestry (214 pre-treatment invasive EOC cases and 214 controls) between the ages of 27 and 91 enrolled in the Mayo Clinic Ovarian Cancer Study. Of the EOC cases $(n=214), 114$ had tumors of serous histology (66\%), 49 tumors were endometriod (23\%), 13 were clear cell (6\%), 5 were mucinous (2\%) and 6 (3\%) were other/unknown. Further information on clinical, lifestyle, and demographic characteristics of the study population is provided in Table 1 and Additional file 4. In general, baseline characteristics of EOC cases versus controls were similar to those estimated based on previous studies of known risk factors (Additional file 4).

\section{Inferred immune cell subsets differ between ovarian cases and controls}

As DNAm was profiled using genomic DNA from wholeblood, which is comprised of genetic substrate from various leukocyte subtypes, the methylation signatures in our study population represent the aggregate methylation profile across a complex cellular landscape. To examine the predicted differences in the major leukocyte components of whole blood (i.e., B cells, natural killer (NK) cells, CD8+ T lymphocytes, CD4+ T lymphocytes, monocytes, and granulocytes) we utilized the cell mixture methodology of Houseman et al. [13]. This method uses a reference panel consisting of the DNAm signatures of isolated leukocyte subtypes to deconvolve the distribution of white blood cell types when DNAm is profiled in whole blood. As noted in Figure 2A, several of the estimated cell type proportions showed statistically significant $(p<0.05)$ differences between EOC cases and controls. More importantly, the relationship between cell type and EOC status was highly consistent between both batches of samples within our study population and also with the results reported in a prior publication [13], which consisted of the application of the cell mixture methodology to an independent study population of $n=131$ pretreatment EOC cases and $n=274$ controls [12]. In particular, granulocyte fractions were higher in EOC cases, while CD8+ T lymphocyte and CD4+ $\mathrm{T}$ lymphocyte, and, to a lesser extent $\mathrm{B}$ cell and NK cell fractions, were lower in EOC cases compared to controls.

These results combined with known methylation differences by cell type suggest that it is critical and feasible to adjust for the underlying distribution of cell types when investigating the relationship between DNAm and EOC case/control status. In particular, Figure 2 shows the epigenome-wide association between DNAm and EOC status (Batch 1 and 2 samples combined) before (Figure 2B,D) and after (Figure 2C,E) adjustment for the estimated cell type proportions, and demonstrates a substantial reduction in the number of differentially methylated CpGs by EOC case/control status upon adjustment.

\section{Identifying CpG dinucleotides that mediate genetic risk for EOC}

Case-control studies focused on the identification of patterns of differential DNAm in the context of disease phenotypes are limited by their retrospective nature and, therefore, are unable to discriminate between patterns that are a consequence of the disease and those that are mediators of disease risk. To filter out consequential epigenetic marks in an attempt to understand biology related to the cause of EOC, we adopted the framework described by Liu et al. [16] for identifying epigenetic marks that are candidate mediators of genetic risk for EOC. To identify instances in which genetic variation influences risk for EOC by regulating CpG-specific methylation patterns we performed a three-step filtering procedure followed by the Causal Inference Test (CIT) [45]. In the first filtering step, we conducted an epigenome-wide association study (EWAS) to identify CpGs differentially methylated by EOC case/control status. Using a series of linear regression models that were adjusted the estimated cell-type proportions and other key covariates, we found 1,993 out of 25,926 (7.7\%) CpGs were associated with EOC case/control status after controlling for multiple comparisons (FDR, $q<0.05)$, step 1 Figure $1 \mathrm{C}$, Additional 
Table 1 Clinical characteristics for the study population

\begin{tabular}{llll}
\hline & $\begin{array}{l}\text { Batch 1 } \\
(\mathbf{N}=\mathbf{1 3 2})\end{array}$ & $\begin{array}{l}\text { Batch 2 } \\
\mathbf{( N = 2 9 6 )}\end{array}$ & $\begin{array}{l}\text { Total } \\
(\mathbf{N}=\mathbf{4 2 8})\end{array}$ \\
\hline $\begin{array}{l}\text { EOC status } \\
\text { Control }\end{array}$ & $66(50.0 \%)$ & $148(50.0 \%)$ & $214(50.0 \%)$ \\
Case & $66(50.0 \%)$ & $148(50.0 \%)$ & $214(50.0 \%)$ \\
\hline Age & & & \\
$\quad$ Mean (SD) & $60(12)$ & $63(13)$ & $62(13)$ \\
Median & 61 & 65 & 64 \\
Q1, Q3 & 50,69 & 54,73 & 52,72 \\
Range & $(33-82)$ & $(27-91)$ & $(27-91)$ \\
\hline Year enrolled & & & \\
$1999-2002$ & $18(13.6 \%)$ & $164(55.4 \%)$ & $182(42.5 \%)$ \\
2003 & $10(7.6 \%)$ & $36(12.2 \%)$ & $46(10.7 \%)$ \\
2004 & $41(31.1 \%)$ & $33(11.1 \%)$ & $74(17.3 \%)$ \\
2005 & $25(18.9 \%)$ & $23(7.8 \%)$ & $48(11.2 \%)$ \\
$2006-2007$ & $38(28.8 \%)$ & $40(13.5 \%)$ & $78(18.2 \%)$ \\
\hline
\end{tabular}

\begin{tabular}{llll}
\hline Parity, number of births & & & \\
Nulliparous & $24(18.2 \%)$ & $42(14.2 \%)$ & $66(15.4 \%)$ \\
$1-2,<=20$ yrs & $7(5.3 \%)$ & $14(4.7 \%)$ & $21(4.9 \%)$ \\
$1-2,>20$ yrs & $31(23.5 \%)$ & $84(28.4 \%)$ & $115(26.9 \%)$ \\
$3+,<=20$ yrs & $28(21.2 \%)$ & $47(15.9 \%)$ & $75(17.5 \%)$ \\
$3+,>20$ yrs & $40(30.3 \%)$ & $95(32.1 \%)$ & $135(31.5 \%)$ \\
\hline
\end{tabular}

\begin{tabular}{llll}
\hline Smoking status (current) & & & \\
No & $120(90.9 \%)$ & $259(87.5 \%)$ & $379(88.6 \%)$
\end{tabular}

\begin{tabular}{llll} 
Yes & $10(7.6 \%)$ & $19(6.4 \%)$ & $29(6.8 \%)$ \\
\hline State & & & \\
Other & $68(51.5 \%)$ & $120(40.5 \%)$ & $188(43.9 \%)$ \\
Minnesota & $64(48.5 \%)$ & $176(59.5 \%)$ & $240(56.1 \%)$ \\
\hline
\end{tabular}

Alcohol consumption

\begin{tabular}{llll} 
Never & $32(24.2 \%)$ & $71(24.0 \%)$ & $103(24.1 \%)$ \\
Current & $78(59.1 \%)$ & $152(51.4 \%)$ & $230(53.7 \%)$ \\
Former & $18(13.6 \%)$ & $47(15.9 \%)$ & $65(15.2 \%)$ \\
\hline $\begin{array}{l}\text { Histology } \\
\text { Serous }\end{array}$ & $43(65.2 \%)$ & $98(66.2 \%)$ & $141(65.9 \%)$ \\
Mucinous & $1(1.5 \%)$ & $4(2.7 \%)$ & $5(2.3 \%)$ \\
Endometrioid & $16(24.2 \%)$ & $33(22.3 \%)$ & $49(22.9 \%)$ \\
Clear Cell & $4(6.1 \%)$ & $9(6.1 \%)$ & $13(6.1 \%)$ \\
Other & $2(3.0 \%)$ & $4(2.7 \%)$ & $6(2.8 \%)$ \\
\hline Grade & & & \\
Grade 1 & $1(1.6 \%)$ & $9(6.1 \%)$ & $10(4.7 \%)$ \\
Grade 2 & $10(15.6 \%)$ & $21(14.2 \%)$ & $31(14.6 \%)$ \\
Grade 3 & $32(50.0 \%)$ & $79(53.4 \%)$ & $111(52.4 \%)$ \\
Grade 4 & $21(32.8 \%)$ & $39(26.4 \%)$ & $60(28.3 \%)$
\end{tabular}

Table 1 Clinical characteristics for the study population (Continued)

\begin{tabular}{rlll}
\hline Stage & & & \\
Stage 1 & $11(16.7 \%)$ & $29(19.6 \%)$ & $40(18.7 \%)$ \\
Stage 2 & $3(4.5 \%)$ & $11(7.4 \%)$ & $14(6.5 \%)$ \\
Stage 3 & $43(65.2 \%)$ & $84(56.8 \%)$ & $127(59.3 \%)$ \\
Stage 4 & $9(13.6 \%)$ & $24(16.2 \%)$ & $33(15.4 \%)$ \\
\hline
\end{tabular}

file 5). As partial validation of these results, we examined the methylation of these 1,993 CpG loci in an independent study population [12]. Our validation analysis revealed that 1,603 out of $1,993 \mathrm{CpG}$ loci $(80 \%)$ were significantly differentially methylated (FDR; $q<0.05$ ), and of these 1,603 loci, 94\% exhibited the same direction of association (i.e., hyper versus hypomethylated) compared to the results obtained from our study population (Additional file 5). Similar to the models fit to our study population, models fit to the independent data were adjusted for the estimated distribution of cell types and subject age; however smoking status, alcohol consumption, parity and population substructure variables were not available in these data and therefore could not be used for adjustment.

In an attempt to identify CpGs where methylation might be genetically influenced, we performed a genome-wide SNP association analysis for each of the 1,993 CpG loci that were differentially methylated between EOC cases and controls. Fitting an allelic dosage model to each of these CpGs and each of 503,502 SNPs, we identified 427 CpG-SNP pairs with genome-wide statistical significance (FDR; $q<0.10$ ) (step 2 Figure 1C, Additional file 6). These 427 CpG-SNP pairs constituted 377 unique SNPs and 185 unique $\mathrm{CpGs}$, and these $\mathrm{CpG}$ loci were disproportionately located in CpG islands (Fisher's Exact; $p=0.017$ ); CpGdense regions present in the promoters of $50 \%-70 \%$ of human genes. Nonetheless, it is still possible that the differential patterns of methylation observed for these $185 \mathrm{CpGs}$ are a consequence of EOC or independently acted on by genotype. To address this concern, we next examined the association between the 377 unique SNPs and EOC status. Of the 377 SNPs, we identified 28 that were significantly associated with EOC status at $P<0.05$ (Additional file 7). These 28 SNPs form 28 CpG-SNP pairs with 19 unique CpGs (step 3 Figure 1C). Implementing the CIT test, we found that the SNP association with EOC was attenuated upon adjustment for methylation for 17 of the 28 CpG-SNP pairs (61\%), suggesting mediation (Figure 3). These $17 \mathrm{CpG-SNP}$ pairs constituted 13 unique CpGs and 17 unique SNPs and represent potential methylation-mediated relationships between genotype and EOC risk (step 4 Figure 1C, Table 2). Information regarding the genomic location and additional annotation for these CpGs and SNPs is provided in Additional files 8 and 9, respectively. 


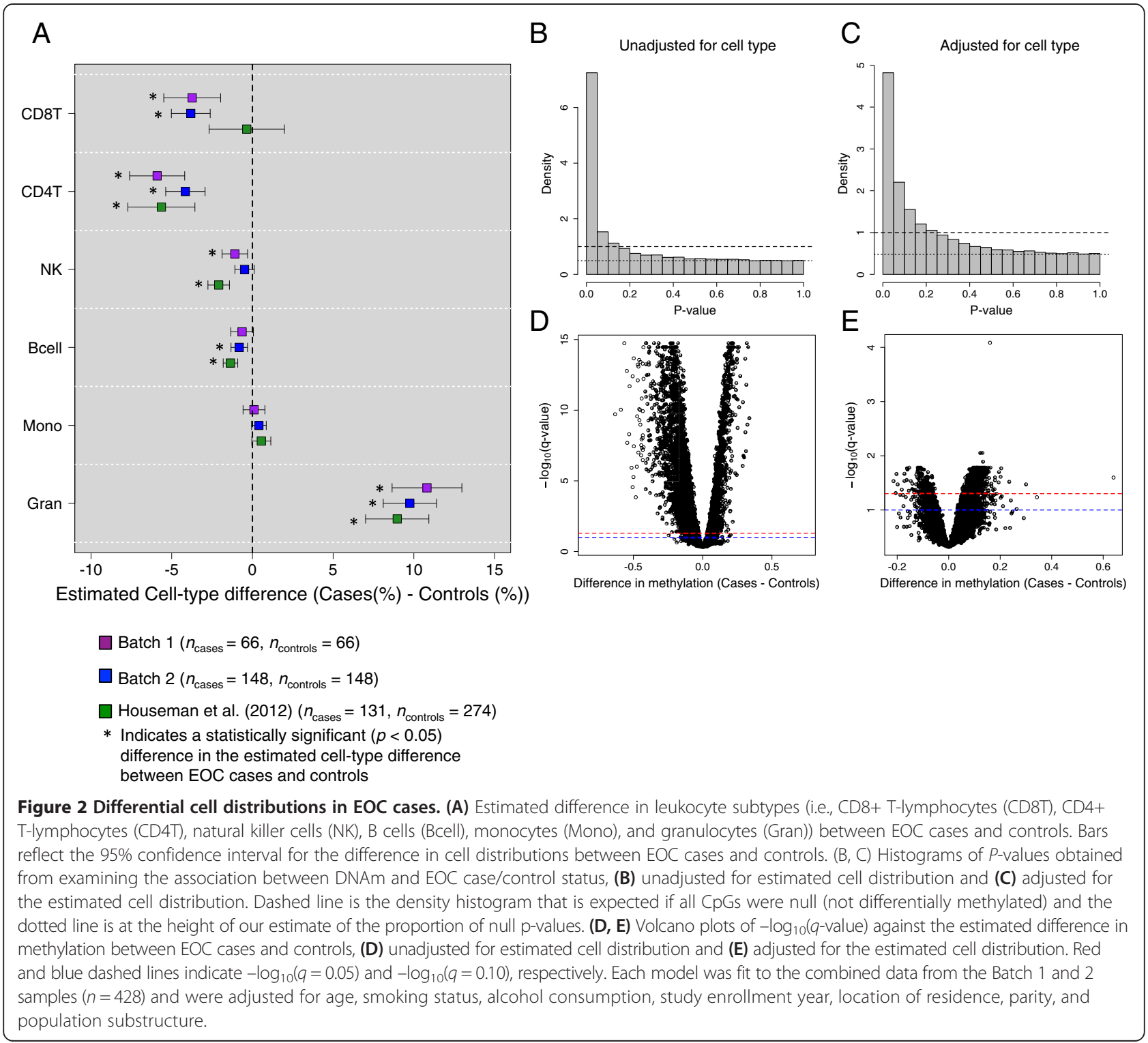

Examining the linkage-disequalibrium (LD) structure among the 17 unique SNPs identified in our analysis showed that most of the identified SNPs were uncorrelated at $\mathrm{r}^{2}<0.05$, with the exception of rs1250220 and rs1250252 located in an intragenic region on chromosome 2q35 and rs6754181, rs2631958, rs2254066, located in an intron region associated with $A L K$ (anaplastic lymphoma receptor tyrosine kinase) (Additional file 10). The 17 CpG-SNP pairs highlight $13 \mathrm{CpG}$ loci; all loci correlate with a single SNP or SNP cluster, although cg10636246 located near AIM2 demonstrated an association with two independent SNPs with different genomic locations ( $r$ 11120596 $\left(p=8 \times 10^{-7}\right)$ and rs460380 $\left.\left(p=5 \times 10^{-7}\right)\right)$ (Table 2).

As differences in tumor DNAm, epidemiologic risk factors, genetic variants, and precursor tissues are known to exist between the major EOC histologies (serous, mucinous, endometrioid, and clear cell) [4], we evaluated whether the 13 unique methylation mediators exhibited consistent patterns of methylation in a ca se-only analysis across EOC subtypes. With the exception of cg25553916 located in the promoter region of FLJ22318 (required for meiotic nuclear division 5 homolog B), which showed increased methylation in mucinous cases $(p=0.006)$, the methylation levels of the 12 remaining $\mathrm{CpG}$ loci were not statistically significant different across the histologies of EOC (Additional file 11). Interestingly, this locus was the only one among the 13 that did not exhibit the same direction of association with EOC comparing our results to those from the Teschendorff et al. [12] data (Table 2 and Additional file 5). 


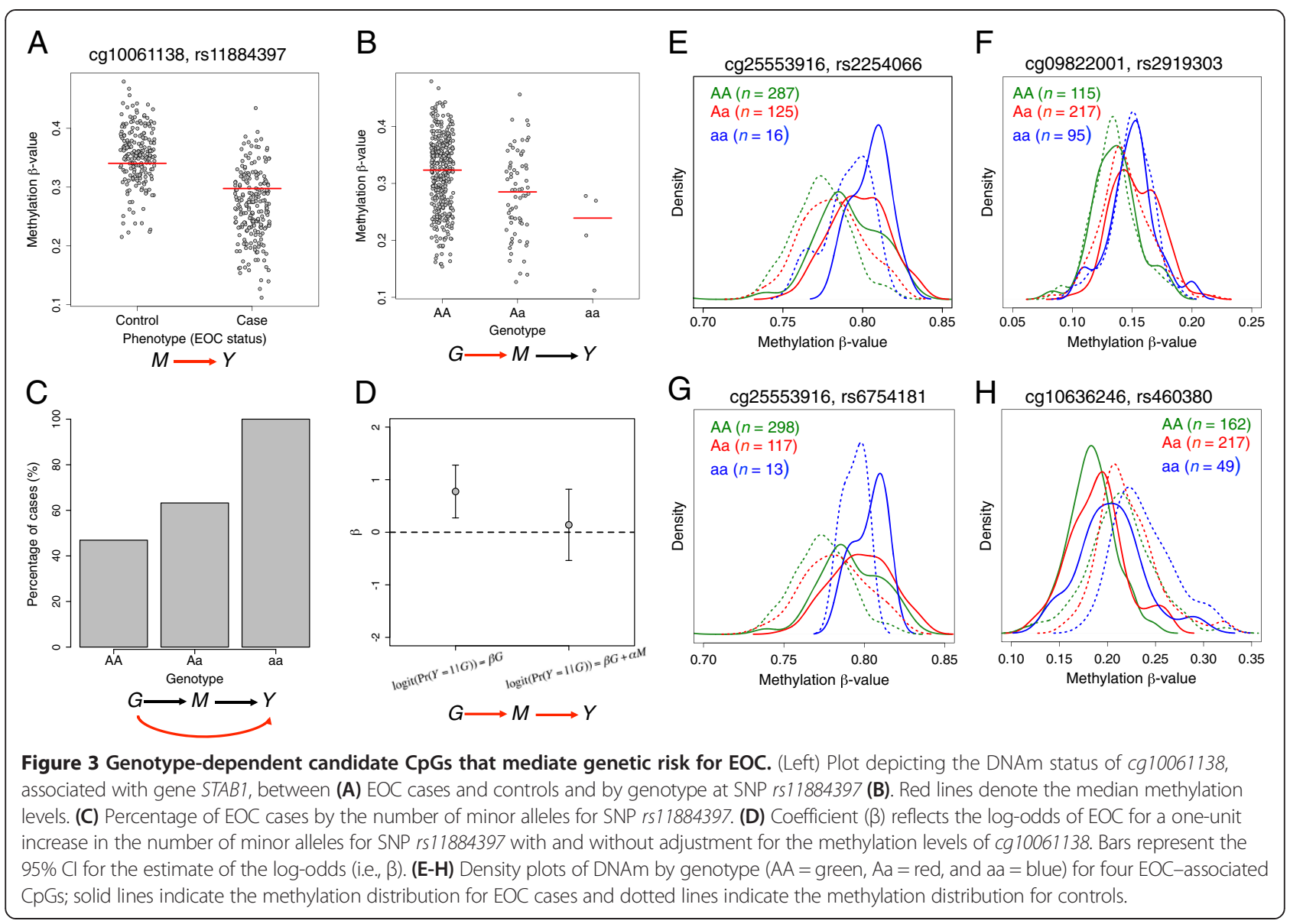

\section{Discussion}

Attempts aimed at distinguishing causal methylation marks from those that are merely a consequence of disease are critical for elucidating the biological mechanisms underlying this disease. Previous analyses of genetic regulators of methylation and expression levels have revealed three-way causal relationships, where the prevailing model is one in which genetic variation influences methylation that in turn influences expression levels. The idea that DNA methylation levels at specific loci are under genetic control has gained traction in recent years, bolstered by the results obtained from comparing patterns of DNA methylation between monozygotic and dizygotic twins [47]. Here, we aimed to leverage these findings in an attempt to filter out epigenetic marks resulting from disease, focusing our attention on the identification of epigenetic marks that are potential mediators of genetic risk for EOC. Not only are such analyses critical for our understanding of EOC pathogenesis, but the genotype-methylation markers identified through such efforts may further enhance the growing library of risk-associated biomarkers for EOC.

Associations between genetic variation with expression and methylation levels have been identified in several organisms [48,49] and tissue types [50]. While recent work has demonstrated both local (cis) and distal (trans) associations of genetic variation with methylation levels [51-53], little is known about the precise biological mechanisms by which genetic variants modify DNA methylation. All of the methylation-genotype pairs identified in our analysis indicated trans regulation, or distant regulation effects. Although none of the 17 SNPs identified in our analysis have been previously identified as reaching genome-wide statistical significance in GWAS of ovarian cancer, of particular importance was the identification of $A L K$ as a potential regulator of CpG-specific DNAm and genetic risk marker for EOC. This gene encodes a receptor tyrosine kinase belonging to the insulin receptor superfamily, and has been found to be rearranged, mutated, or amplified in a series of human cancer tumorigenesis [54-56]. Recent work has demonstrated methylation induced silencing of IL-2R $\gamma$ expression in in T-cell lymphoma cells expressing NPM-ALK kinase [57], which originates from fusion of the nucleophosmin (NPM) and the membrane receptor anaplastic lymphoma kinase genes. IL-2R $\gamma$ is shared by receptors for several cytokines that play key roles in the maturation and growth of normal CD4+ T lymphocytes and other immune cells. Thus, it is possible that genetic variation in $A L K$ contributes to epigenetic modifications 
Table 2 CpG sites that were identified as potential mediators of genetic risk for EOC $(C I T p<0.05)$

\begin{tabular}{|c|c|c|c|c|c|c|c|c|c|c|c|}
\hline \multirow[b]{2}{*}{ CpG } & \multicolumn{5}{|c|}{ CpGs associated with EOC } & \multicolumn{6}{|c|}{ SNPs associated with CpGs } \\
\hline & Meth diff & $\begin{array}{l}\text { P-value } \\
\text { (M vs Y) }\end{array}$ & Gene & $\mathrm{Chr}$ & Pos & SNP & $\mathrm{Chr}$ & Pos & $\begin{array}{l}\text { P-value } \\
\text { (G vs M) }\end{array}$ & $\begin{array}{l}\text { P-value } \\
\text { (G vs Y) }\end{array}$ & $\begin{array}{l}\text { P-value } \\
\text { (CIT) }\end{array}$ \\
\hline cg03718677 & -0.08 & 4.60E-03 & TMOD4 & 1 & 149414890 & rs1250220 & 2 & 216320050 & $2.30 \mathrm{E}-07$ & 0.023 & 0.023 \\
\hline cg03718677 & -0.08 & 4.60E-03 & TMOD4 & 1 & 149414890 & rs1250252 & 2 & 216313591 & $1.70 \mathrm{E}-07$ & 0.029 & 0.029 \\
\hline cg09822001 & 0.11 & $5.50 \mathrm{E}-04$ & $A P O A 1 B P$ & 1 & 154827958 & rs2919303 & 8 & 62214493 & $1.50 \mathrm{E}-07$ & 0.027 & 0.027 \\
\hline $\operatorname{cg} 10636246$ & -0.11 & 2.70E-04 & AIM2 & 1 & 157313597 & rs11120596 & 1 & 215850817 & $7.60 \mathrm{E}-07$ & 0.002 & 0.002 \\
\hline $\operatorname{cg} 10636246$ & -0.11 & 2.70E-04 & AIM2 & 1 & 157313597 & rs460380 & 21 & 46028864 & 5.30E-07 & 0.01 & 0.01 \\
\hline cg13721560 & 0.07 & 7.90E-03 & $\angle R P P R C$ & 2 & 44076072 & rs2289840 & 3 & 124181739 & $9.40 \mathrm{E}-08$ & 0.041 & 0.041 \\
\hline cg10061138 & -0.09 & 8.30E-04 & STAB1 & 3 & 52504125 & rs11884397 & 2 & 23885388 & $3.10 \mathrm{E}-07$ & 0.002 & 0.002 \\
\hline cg25086702 & 0.1 & 1.70E-03 & HMGB2 & 4 & 174492134 & rs11210834 & 1 & 43787437 & 7.10E-08 & 0.048 & 0.048 \\
\hline cg24136586 & 0.1 & 5.50E-03 & ATG10 & 5 & 81304122 & rs3800524 & 6 & 168302015 & $1.80 \mathrm{E}-06$ & 0.034 & 0.034 \\
\hline cg01495509 & 0.08 & 5.30E-03 & KCNMB1 & 5 & 169748956 & rs4457945 & 15 & 54608127 & 2.20E-08 & 0.019 & 0.019 \\
\hline cg25553916 & 0.06 & $6.90 \mathrm{E}-04$ & FLL22318 & 5 & 177491361 & rs2254066 & 2 & 29889019 & $2.60 \mathrm{E}-07$ & 0.003 & 0.003 \\
\hline cg25553916 & 0.06 & $6.90 \mathrm{E}-04$ & FLJ22318 & 5 & 177491361 & rs2631958 & 2 & 29958841 & 7.10E-07 & 0.009 & 0.009 \\
\hline cg25553916 & 0.06 & $6.90 \mathrm{E}-04$ & FLJ22318 & 5 & 177491361 & rs6754181 & 2 & 29966380 & $3.10 \mathrm{E}-07$ & 0.003 & 0.003 \\
\hline cg19436567 & 0.12 & 1.50E-04 & $A R I D 1 B$ & 6 & 157141067 & rs12362925 & 11 & 16875419 & $7.80 \mathrm{E}-08$ & 0.029 & 0.029 \\
\hline cg08142684 & 0.13 & 2.70E-04 & TCP1 & 6 & 160129858 & rs9792311 & 8 & 123940585 & $7.40 \mathrm{E}-07$ & 0.023 & 0.023 \\
\hline cg05109049 & 0.09 & $5.30 \mathrm{E}-03$ & $E V I 2 B$ & 17 & 26665459 & rs2294405 & 6 & 99292136 & $1.60 \mathrm{E}-06$ & 0.013 & 0.013 \\
\hline cg00021527 & 0.09 & $5.20 \mathrm{E}-03$ & TAF15 & 17 & 31160293 & rs10488500 & 7 & 76841259 & 2.50E-07 & 0.046 & 0.046 \\
\hline
\end{tabular}

M: Methylation, G: Genotype, Y: EOC status.

Methdiff: Difference in the methylation M-value between EOC cases minus controls, adjusted for the estimated distribution of cell types, age, smoking status, alcohol consumption, study enrollment year, location of residence, parity and age at first birth, and the first principal component representing within-European population sub-structure.

Gene: Denotes the nearest gene for the given CpG site.

that alter the normal functioning of immune cells, however the exact biological mechanisms by which $A L K$ exerts an influence on DNA methylation is unclear.

It is also compelling that the predicted distribution of immune cell subsets in our data, which showed increased myeloid derived cell types (e.g., monocytes and granulocytes) and decreased lymphocytes (e.g., CD8+ T lymphocytes, CD4+ T lymphocytes, B cells, and natural killer cells) between EOC cases and controls, mimicked the results obtained when applying the cell mixture methodology to an independent study population. The relationship between predicted cell type distributions and cancer status are consistent with previous literature, where it has been demonstrated that EOC cases have decreased B and T-lymphocyte fractions [41-43] and increases in neutrophil granulocytes [43]. While modest variation was observed in the estimated cell type differences between EOC cases and controls between the different study populations, particularly for CD8+ Tlymphocytes, this is not entirely unexpected given differences in the distribution of ovarian cancer histological subtypes between the study populations (Table 1; Teschendorff et al. [12]) and that variation in host immune responses to EOC has been shown to vary by histological subtype [58].
Our analysis identified cg25086702 as a potential mediator of genetic risk for EOC. This particular locus resides in CPG island region located in HMGB2 (high mobility group protein 2) and was found to be hypermethylated in EOC in the present analysis $(\beta=0.10,95 \%$ CI $[0.04,0.16])$. High mobility group box $(H M G B)$ proteins are ubiquitous, abundant nuclear proteins with diverse functions in the cell. $H M G B 1$ and $H M G B 2$ are the main members of the $H M G B$ protein family and their overexpression has been observed in numerous human malignancies, including hepatocellular [59], skin squamous cell [60], prostate [61], gastrointestinal [62,63] breast [64,65], and bladder carcinomas [66]. Additionally, a recent report demonstrated increased expression of HMGB2 in invasive EOC tumors compared to EOC tumors with low malignancy potential [67]. However, many of the results demonstrating HMGB2 overexpression were based on measurements derived from tumor tissue, and far less is known about the implications of dysregulated HMGB2 expression in peripheral blood leukocytes and its role in cancer risk. While it is possible that our observation of increased $\mathrm{CpG}$ island methylation of $H M G B 2$ in EOC cases is due to the methylation signature arising from circulating tumor cells (CTCs), this is unlikely as CTCs would be expected to comprise 
a small fraction of the total cells used in assessing DNAm [68], and thus contribute insignificantly to the overall methylation signatures analyzed. Also, given the role CpG island hypermethylation on gene silencing and the numerous reports of $H M G B$ overexpression in tumor tissue, we might expect to see the opposite results (i.e., $\mathrm{CpG}$ island hypomethlation of $H M G B 2$ ) if in fact, CTCs were driving force behind the methylation signals detected here. An alternative explanation for these findings is motivated by the role of $H M G B 1$, a closely related gene, in immune response in adult peripheral blood. $H M G B 1$ recruits inflammatory cells and activates innate immune cells. Furthermore, after being secreted by activated macrophages or its release from necrotic cells, HMGB1 regulates adaptive immunity [69-71]. Thus, CpG island hypermethylation induced silencing of $H M G B 1$ and possibly other $H M G B$ genes, may compromise the immune system, promoting tumor development and progression.

Our analysis also identified cg05109049 $(\beta=0.09,95 \%$ CI $[0.03,0.16]$ ), associated with EVI2B (Ecotropic Viral Integration Site 2B Protein), as a potential mediator of genetic risk for EOC. EVI $2 B$ is expressed in peripheral blood mononuclear cells, fibroblasts, and bone marrow and blood-based overexpression of this gene was recently reported in postoperative relapse of colorectal cancer [72]. Another notable discovery from our analysis was hypermethylation of $\operatorname{cg} 00021527$ ( $\beta=0.09,95 \% \mathrm{CI}[0.03,0.15]$ ), residing in a CpG Island region located in the gene TAF15 (TATA box-binding protein-associated factor $2 \mathrm{~N} 68 \mathrm{kDa}$ ), which together with FUS (fused in sarcoma) and EWS (Ewing sarcoma breakpoint 1), constitute the FET protein family. The FET-proteins are involved in transcriptional regulation and RNA processing, and FET-gene deregulation is associated with development of cancer. In particular, a recent report demonstrated that TAF15 knockdown affects the expression of a large subset of genes, including many involved in cell cycle and cell death [73]. Together, these findings highlight the biological relevance of the methylation sites identified in our investigation and their potential role in the pathogenesis of EOC.

There are several noteworthy limitations to the present study. First, the relatively small sample size and large number of genotype/methylation markers, reduces our statistical power for detecting genotype/methylation associations. To address the burden of multiple comparisons arising from the large number of genotype/methylation markers, we employed an analytical strategy that is based on a series of filtering steps, resulting in many fewer overall tests than an analysis considering all possible genotype, methylation, and phenotype combinations. Further, while Illumina Infinium HumanMethylation27 BeadChip provides an efficient solution for surveying genome-wide DNA methylation profiles, the lower coverage and scope of this array compared to more recent array technologies, e.g., Illumina Infinium HumanMethylation450 BeadChip, may have limited our ability for detecting methylation mediators of EOC genetic risk.

With regard to our sample size, efforts to replicate the analysis described here using a larger group of study subjects as both a validation of our existing results, and to identify additional methylation sites that mediate genetic risk for EOC is ongoing research by our group. Moreover, we additionally evaluated the results of our EWAS analysis using a previously published data set, which consisted of whole-blood derived methylation data assayed using the same array technology - collected from pre-treatment EOC cases and controls. Unlike our data, genotypic information was not available on those subjects, preventing a complete validation of the genotype-me thylation pairs identified here. However, the fact that 12 of the 13 (92\%) CpGs representing potential methylationmediated relationships between genotype and EOC risk demonstrated the same direction of association with EOC status is encouraging and serves as motivation for the continued and future study of these markers.

A second consideration of this work involves the potential for confounding based on interpersonal variability the distribution of cell types used in assessing DNA methylation. While previous reports involving bloodbased assessment of DNA methylation have controlled for cell mixture using complete-blood cell count (CBC) measurements $[74,75]$, such measurements are not capable of distinguishing between different lymphocyte subtypes and may be an oversimplification of the complexity and variability in circulating immune cells. Here, we employed a recently developed statistical method for predicting the distribution of the major leukocyte components of whole blood, followed by their inclusion as additional covariates in our methylation association analyses. It should be noted that the cell type predictions obtained using this approach are themselves estimates and therefore subject to uncertainty. Computationally efficient statistical approaches that facilitate the propagation of this uncertainty into locus-specific differential methylation analyses are urgently needed and represent an opportunity for future methodological work.

A limitation of EWAS aimed toward understanding the molecular basis of complex phenotypes over conventional GWAS, is that the methylation sites identified from EWAS may be a consequence of the disease or due to treatment, rather than true biomarkers of disease risk. We attempted to address this limitation by focusing our analysis on pre-treatment EOC cases and through the implementation of a statistical mediation framework that was recently shown to be an effective tool in the analysis of data arising from EWAS [16]. We do however note that in focusing on the identification of candidate methylation mediators of EOC genetic risk, it is possible, and likely, 
that other potentially "causal" epigenetic marks were missed using our analytical strategy. Along these lines, there is an urgent need for studies involving the investigation of prospectively collected methylation profiles and subsequent risk of EOC, such as that carried out in a recent study of breast cancer risk [76]. It should be acknowledged that, as in all case-control studies, it is not possible to establish causality on the basis of purely retrospective observational data. With this in mind, our findings can be viewed as a basis for hypotheses, providing a starting point for future mechanistic studies and studies focused on their validation in independent study populations.

\section{Conclusions}

Overall, this study contributes to the growing archive of integrative genomics studies by exploring the relationship between genetics and epigenetics as they relate to EOC risk. Our analysis identified $17 \mathrm{CpG} / \mathrm{SNP}$ pairs, comprising 13 unique CpGs and 17 unique SNPs, which represent potential methylation-mediated relationships between genotype and EOC risk. These findings provide additional insight into EOC etiology and may serve as novel biomarkers for EOC susceptibility. Future work is needed to independently validate the genotype-methylation markers discovered here and to elucidate their functional role.

\section{Additional files}

Additional file 1: Table S1. P-values based on examining the association between the top 3 principal components and covariate information.

Additional file 2: Figure S1. Plot of the first two principal components computed from the raw DNA methylation data. Black points indicate samples from Batch $1(n=132)$ and red points indicate samples from Batch $2(n=296)$.

Additional file 3: Table S2. Pyrosequencing methylation assay and correlation with Illumina $27 \mathrm{~K}$ methylation beta values.

Additional file 4 Table S3. Information for the 1,993 differentially methylated CPGs between EOC cases and controls.

Additional file 5: Table S4. Association of covariates with case/control status.

Additional file 6: Table S5. Statistically significant CpG/SNP pairs obtained from examining the association between methylation and genotype. Results obtained for non-statistically significant CpG/SNP associations are available from the authors upon request.

Additional file 7: Table S6. Information for the 28 SNPs that were significantly associated with EOC case/control status.

Additional file 8: Table S7. Information for the 13 CpGs that were identified as potential methylation-mediated relationships between genotype and EOC risk

Additional file 9: Table S8. Information for the 17 SNPs that were identified as potential methylation-mediated relationships between genotype and EOC risk

Additional file 10: Figure S2. LD-plots for the 17 unique SNPs identified in the mediation analysis among (A) EOC cases and (B) controls.

Additional file 11: Figure S3. Methylation levels of the 13 unique $\mathrm{CpGs}$, identified as potential mediators of genetic risk for EOC, across the various EOC histologies. HS (high-grade serous), LS (low-grade serous), $\mathrm{M}$ (mucinous), $\mathrm{E}$ (endometriod), and C (clear cell).

\section{Abbreviations}

EWAS: Epigenome-wide association study; EOC: Epithelial ovarian cancer; CTCs: Circulating tumor cells; LD: Linkage disequilibrium; CIT: Causal inference test; DNAm: DNA methylation; PCA: Principal components analysis.

\section{Competing interests}

The authors declare that they have no competing interests.

\section{Authors' contributions}

DK carried out the statistical analysis and drafted the manuscript. PC, ML, and SA contributed to the preprocessing and quality control of genomic data used in this investigation, as well as the preparation of the manuscript $M C, J C, M B, T S$, and JMC provided assistance in the interpretation of the results and helped in the manuscript preparation. BF and EG conceived of the study, and participated in its design and coordination and helped to draft the manuscript. All authors have read and approve the final manuscript.

\section{Acknowledgements}

The preparation of this manuscript, data collection and processing, and the analysis implemented in this examination was supported by the National Institute of Health grants, U19 CA148112 R01 CA122443 R01 CA114343.

\section{Author details}

'Department of Biostatistics, University of Kansas Medical Center, 3901 Rainbow Blvd, Kansas City, KS 66160, USA. ²Department of Cancer Biology, University of Kansas Medical Center, Kansas City, KS 66160, USA. ${ }^{3}$ Department of Health Sciences Research, Mayo Clinic, Rochester, MN 55905, USA.

${ }^{4}$ Laboratory Medicine and Pathology, Mayo Clinic, Rochester, MN 55905, USA.

${ }^{5}$ Medical Oncology, Mayo Clinic, Rochester, MN 55905, USA. ${ }^{6}$ Office of the

Director, Moffitt Cancer Center, Tampa, FL 33612, USA.

Received: 10 October 2013 Accepted: 22 January 2014

Published: 30 January 2014

\section{References}

1. Siegel R, Naishadham D, Jemal A: Cancer statistics, 2013. CA Cancer J Clin 2013, 63(1):11-30.

2. Bodurka-Bevers D, Sun CC, Gershenson DM: Pharmacoeconomic considerations in treating ovarian cancer. Pharmacoeconomics 2000 17(2):133-150.

3. Mariotto $A B$, Yabroff KR, Shao Y, Feuer EJ, Brown ML: Projections of the cost of cancer care in the United States: 2010-2020. J Natl Cancer Inst 2011, 103(2):117-128.

4. Cicek MS, Koestler DC, Fridley BL, Kalli KR, Armasu SM, Larson MC, Wang C, Winham SJ, Vierkant RA, Rider DN, et al: Epigenome-wide ovarian cancer analysis identifies a methylation profile differentiating clear-cell histology with epigenetic silencing of the HERG K + channel. Hum Mol Genet 2013, 22(15):3038-3047.

5. Bauerschlag DO, Ammerpohl O, Brautigam K, Schem C, Lin Q, Weigel MT, Hilpert F, Arnold N, Maass N, Meinhold-Heerlein I, et al: Progression-free survival in ovarian cancer is reflected in epigenetic DNA methylation profiles. Oncology 2011, 80(1-2):12-20.

6. Watts GS, Futscher BW, Holtan N, Degeest K, Domann FE, Rose SL: DNA methylation changes in ovarian cancer are cumulative with disease progression and identify tumor stage. BMC Med Genomics 2008, 1:47

7. Shen H, Fridley BL, Song H, Lawrenson K, Cunningham JM, Ramus SJ, Cicek MS, Tyrer J, Stram D, Larson MC, et al: Epigenetic analysis leads to identification of HNF1B as a subtype-specific susceptibility gene for ovarian cancer. Nat Commun 2013, 4:1628.

8. Langevin SM, Koestler DC, Christensen BC, Butler RA, Wiencke JK, Nelson $\mathrm{HH}$, Houseman EA, Marsit CJ, Kelsey KT: Peripheral blood DNA methylation profiles are indicative of head and neck squamous cell carcinoma: an epigenome-wide association study. Epigenetics 2012, 7(3):291-299.

9. Marsit CJ, Koestler DC, Christensen BC, Karagas MR, Houseman EA, Kelsey KT: DNA methylation array analysis identifies profiles of blood-derived DNA methylation associated with bladder cancer. J Clin Oncol 2011, 29(9):1133-1139.

10. Pedersen $\mathrm{KS}$, Bamlet WR, Oberg AL, de Andrade M, Matsumoto ME, Tang $\mathrm{H}_{\text {, }}$ Thibodeau SN, Petersen GM, Wang L: Leukocyte DNA methylation signature differentiates pancreatic cancer patients from healthy controls. PLoS One 2011, 6(3):e18223 
11. Wang L, Aakre JA, Jiang R, Marks RS, Wu Y, Chen J, Thibodeau SN, Pankratz VS, Yang P: Methylation markers for small cell lung cancer in peripheral blood leukocyte DNA. J Thorac Oncol 2010, 5(6):778-785.

12. Teschendorff AE, Menon U, Gentry-Maharaj A, Ramus SJ, Gayther SA, Apostolidou S, Jones A, Lechner M, Beck S, Jacobs IJ, et al: An epigenetic signature in peripheral blood predicts active ovarian cancer. PLoS One 2009, 4(12):e8274.

13. Houseman EA, Accomando WP, Koestler DC, Christensen BC, Marsit CJ, Nelson $\mathrm{HH}$, Wiencke JK, Kelsey KT: DNA methylation arrays as surrogate measures of cell mixture distribution. BMC Bioinforma 2012, 13(1):86

14. Koestler DC, Marsit CJ, Christensen BC, Accomando W, Langevin SM, Houseman EA, Nelson HH, Karagas MR, Wiencke JK, Kelsey KT: Peripheral blood immune cell methylation profiles are associated with nonhematopoietic cancers. Cancer Epidemiol Biomarkers Prev 2012, 21(8):1293-1302.

15. Reinius LE, Acevedo N, Joerink M, Pershagen G, Dahlen SE, Greco D, Soderhall C, Scheynius A, Kere J: Differential DNA methylation in purified human blood cells: implications for cell lineage and studies on disease susceptibility. PLoS One 2012, 7(7):e41361.

16. Liu Y, Aryee MJ, Padyukov L, Fallin MD, Hesselberg E, Runarsson A, Reinius L, Acevedo N, Taub M, Ronninger M, et al: Epigenome-wide association data implicate DNA methylation as an intermediary of genetic risk in rheumatoid arthritis. Nat Biotechnol 2013, 31(2):142-147.

17. Lin PC, Giannopoulou EG, Park K, Mosquera JM, Sboner A, Tewari AK, Garraway LA, Beltran H, Rubin MA, Elemento O: Epigenomic alterations in localized and advanced prostate cancer. Neoplasia 2013, 15(4):373-383.

18. Baylin SB, Jones PA: A decade of exploring the cancer epigenome biological and translational implications. Nat Rev Cancer 2011, 11(10):726-734.

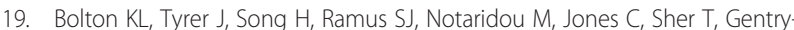
Maharaj A, Wozniak E, Tsai YY, et al: Common variants at 19p13 are associated with susceptibility to ovarian cancer. Nat Genet 2010, 42(10):880-884.

20. Goode EL, Chenevix-Trench G, Song H, Ramus SJ, Notaridou M, Lawrenson K, Widschwendter M, Vierkant RA, Larson MC, Kjaer SK, et al: A genome-wide association study identifies susceptibility loci for ovarian cancer at 2q31 and 8q24. Nat Genet 2010, 42(10):874-879.

21. Song H, Ramus SJ, Tyrer J, Bolton KL, Gentry-Maharaj A, Wozniak E, AntonCulver H, Chang-Claude J, Cramer DW, DiCioccio R, et al: A genome-wide association study identifies a new ovarian cancer susceptibility locus on 9p22.2. Nat Genet 2009, 41(9):996-1000.

22. Pharoah PD, Tsai YY, Ramus SJ, Phelan CM, Goode EL, Lawrenson K, Buckley $M$, Fridley BL, Tyrer JP, Shen H, et al: GWAS meta-analysis and replication identifies three new susceptibility loci for ovarian cancer. Nat Genet 2013, 45(4)):362-370. 370e361-362.

23. Bojesen SE, Pooley KA, Johnatty SE, Beesley J, Michailidou K, Tyrer JP, Edwards SL, Pickett HA, Shen HC, Smart CE, et al: Multiple independent variants at the TERT locus are associated with telomere length and risks of breast and ovarian cancer. Nat Genet 2013, 45(4):371-384. 384e371-372.

24. Permuth-Wey J, Lawrenson K, Shen HC, Velkova A, Tyrer JP, Chen Z, Lin HY, Chen YA, Tsai YY, Qu X, et al: Identification and molecular characterization of a new ovarian cancer susceptibility locus at 17q21.31. Nat Commun 2013, 4:1627.

25. Painter JN, Anderson CA, Nyholt DR, Macgregor S, Lin J, Lee SH, Lambert A, Zhao ZZ, Roseman F, Guo Q, et al: Genome-wide association study identifies a locus at 7p15.2 associated with endometriosis. Nat Genet 2011, 43(1):51-54.

26. Cedar $\mathrm{H}$, Bergman $\mathrm{Y}$ : Programming of DNA methylation patterns. Annu Rev Biochem 2012, 81:97-117

27. Permuth-Wey J, Kim D, Tsai YY, Lin HY, Chen YA, Barnholtz-Sloan J, Birrer MJ, Bloom G, Chanock SJ, Chen Z, et al: LIN28B Polymorphisms influence susceptibility to epithelial ovarian cancer. Cancer Res 2011, 71(11):3896-3903.

28. Chelala C, Khan A, Lemoine NR: SNPnexus: a web database for functional annotation of newly discovered and public domain single nucleotide polymorphisms. Bioinformatics 2009, 25(5):655-661.

29. Dayem Ullah AZ, Lemoine NR, Chelala C: SNPnexus: a web server for functional annotation of novel and publicly known genetic variants (2012 update). Nucleic Acids Res 2012, 40(Web Server issue):W65-W70.

30. Dayem Ullah AZ, Lemoine NR, Chelala C: A practical guide for the functional annotation of genetic variations using SNPnexus. Brief Bioinform 2013, 14(4):437-447.
31. Yang HH, Hu N, Wang C, Ding T, Dunn BK, Goldstein AM, Taylor PR, Lee MP: Influence of genetic background and tissue types on global DNA methylation patterns. PLoS One 2010, 5(2):e9355.

32. Harper KN, Peters BA, Gamble MV: Batch effects and pathway analysis: two potential perils in cancer studies involving DNA methylation array analysis. Cancer Epidemiol Biomarkers Prev 2013, 22(6):1052-1060.

33. Du $P$, Zhang $X$, Huang CC, Jafari N, Kibbe WA, Hou L, Lin SM: Comparison of Beta-value and M-value methods for quantifying methylation levels by microarray analysis. BMC Bioinforma 2010, 11:587.

34. Kuan PF, Wang S, Zhou X, Chu H: A statistical framework for Illumina DNA methylation arrays. Bioinformatics 2010, 26(22):2849-2855.

35. Johnson WE, Li C, Rabinovic A: Adjusting batch effects in microarray expression data using empirical Bayes methods. Biostatistics 2007, 8(1):118-127.

36. Chen C, Grennan K, Badner J, Zhang D, Gershon E, Jin L, Liu C: Removing batch effects in analysis of expression microarray data: an evaluation of six batch adjustment methods. PLoS One 2011, 6(2).

37. Kupfer P, Guthke R, Pohlers D, Huber R, Koczan D, Kinne RW: Batch correction of microarray data substantially improves the identification of genes differentially expressed in rheumatoid arthritis and osteoarthritis. BMC Med Genomics 2012, 5:23.

38. Dedeurwaerder S, Defrance M, Bizet M, Calonne E, Bontempi G, Fuks F: A comprehensive overview of infinium HumanMethylation450 data processing. Brief Bioinform 2013.

39. Morris TJ, Butcher LM, Feber A, Teschendorff AE, Chakravarthy AR, Wojdacz TK, Beck S: ChAMP: 450k chip analysis methylation pipeline. Bioinformatics 2013.

40. Wilhelm-Benartzi CS, Koestler DC, Karagas MR, Flanagan JM, Christensen BC, Kelsey KT, Marsit CJ, Houseman EA, Brown R: Review of processing and analysis methods for DNA methylation array data. Br J Cancer 2013, 109(6):1394-1402.

41. Bishara S, Griffin M, Cargill A, Bali A, Gore ME, Kaye SB, Shepherd JH, Van Trappen PO: Pre-treatment white blood cell subtypes as prognostic indicators in ovarian cancer. Eur J Obstet Gynecol Reprod Biol 2008, 138(1):71-75.

42. Cho H, Hur HW, Kim SW, Kim SH, Kim JH, Kim YT, Lee K: Pre-treatment neutrophil to lymphocyte ratio is elevated in epithelial ovarian cancer and predicts survival after treatment. Cancer Immunol Immunother 2009, 58(1):15-23.

43. den Ouden M, Ubachs JM, Stoot JE, van Wersch JW: Whole blood cell counts and leucocyte differentials in patients with benign or malignant ovarian tumours. Eur J Obstet Gynecol Reprod Biol 1997, 72(1):73-77.

44. Koestler DC, Christensen B, Karagas MR, Marsit CJ, Langevin SM, Kelsey KT, Wiencke JK, Houseman EA: Blood-based profiles of DNA methylation predict the underlying distribution of cell types: a validation analysis. Epigenetics 2013, 8(8)

45. Millstein J, Zhang B, Zhu J, Schadt EE: Disentangling molecular relationships with a causal inference test. BMC Genet 2009, 10:23.

46. Storey JD, Tibshirani R: Statistical significance for genomewide studies. Proc Natl Acad Sci USA 2003, 100(16):9440-9445.

47. Kaminsky ZA, Tang T, Wang SC, Ptak C, Oh GH, Wong AH, Feldcamp LA, Virtanen C, Halfvarson J, Tysk C, et al: DNA methylation profiles in monozygotic and dizygotic twins. Nat Genet 2009, 41(2):240-245.

48. Cookson W, Liang L, Abecasis G, Moffatt M, Lathrop M: Mapping complex disease traits with global gene expression. Nat Rev Genet 2009, 10(3):184-194.

49. Stranger BE, Dermitzakis ET: The genetics of regulatory variation in the human genome. Hum Genomics 2005, 2(2):126-131.

50. Nica AC, Parts L, Glass D, Nisbet J, Barrett A, Sekowska M, Travers M, Potter S, Grundberg E, Small K, et al: The architecture of gene regulatory variation across multiple human tissues: the MuTHER study. PLOS Genet 2011, 7(2):e1002003.

51. Bell JT, Pai AA, Pickrell JK, Gaffney DJ, Pique-Regi R, Degner JF, Gilad Y, Pritchard JK: DNA methylation patterns associate with genetic and gene expression variation in HapMap cell lines. Genome Biol 2011, 12(1):R10.

52. Gibbs JR, van der Brug MP, Hernandez DG, Traynor BJ, Nalls MA, Lai SL, Arepalli S, Dillman A, Rafferty IP, Troncoso J, et al: Abundant quantitative trait loci exist for DNA methylation and gene expression in human brain. PLoS Genet 2010, 6(5):e1000952.

53. Zhang D, Cheng L, Badner JA, Chen C, Chen Q, Luo W, Craig DW, Redman $M$, Gershon ES, Liu C: Genetic control of individual differences in 
gene-specific methylation in human brain. Am J Hum Genet 2010, 86(3):411-419.

54. Janoueix-Lerosey I, Lequin D, Brugieres L, Ribeiro A, de Pontual L, Combaret V, Raynal V, Puisieux A, Schleiermacher G, Pierron G, et al: Somatic and germline activating mutations of the ALK kinase receptor in neuroblastoma. Nature 2008, 455(7215):967-970.

55. Webb TR, Slavish J, George RE, Look AT, Xue L, Jiang Q, Cui X, Rentrop WB, Morris SW: Anaplastic lymphoma kinase: role in cancer pathogenesis and small-molecule inhibitor development for therapy. Expert Rev Anticancer Ther 2009, 9(3):331-356.

56. Wong DW, Leung EL, So KK, Tam IY, Sihoe AD, Cheng LC, Ho KK, Au JS, Chung LP, Pik Wong M: The EML4-ALK fusion gene is involved in various histologic types of lung cancers from nonsmokers with wild-type EGFR and KRAS. Cancer 2009, 115(8):1723-1733.

57. Zhang Q, Wang HY, Liu X, Bhutani G, Kantekure K, Wasik M: IL-2R common gamma-chain is epigenetically silenced by nucleophosphin-anaplastic lymphoma kinase (NPM-ALK) and acts as a tumor suppressor by targeting NPM-ALK. Proc Natl Acad Sci USA 2011, 108(29):11977-82.

58. Milne K, Kobel M, Kalloger SE, Barnes RO, Gao D, Gilks CB, Watson PH, Nelson $\mathrm{BH}$ : Systematic analysis of immune infiltrates in high-grade serous ovarian cancer reveals CD20, FoxP3 and TIA-1 as positive prognostic factors. PLoS One 2009, 4(7):e6412.

59. Kostova N, Zlateva S, Ugrinova I, Pasheva E: The expression of HMGB1 protein and its receptor RAGE in human malignant tumors. Mol Cell Biochem 2010, 337(1-2):251-258.

60. Sharma A, Ray R, Rajeswari MR: Overexpression of high mobility group (HMG) B1 and B2 proteins directly correlates with the progression of squamous cell carcinoma in skin. Cancer Invest 2008, 26(8):843-851.

61. Gnanasekar M, Thirugnanam S, Ramaswamy K: Short hairpin RNA (shRNA) constructs targeting high mobility group box-1 (HMGB1) expression leads to inhibition of prostate cancer cell survival and apoptosis. Int $\rfloor$ Oncol 2009, 34(2):425-431.

62. Song B, Song WG, Li ZJ, Xu ZF, Wang XW, Wang CX, Liu J: Effect of HMGB1 silencing on cell proliferation, invasion and apoptosis of MGC-803 gastric cancer cells. Cell Biochem Funct 2011.

63. Yao X, Zhao G, Yang H, Hong X, Bie L, Liu G: Overexpression of highmobility group box 1 correlates with tumor progression and poor prognosis in human colorectal carcinoma. J Cancer Res Clin Oncol 2010, 136(5):677-684.

64. Jiao Y, Wang HC, Fan SJ: Growth suppression and radiosensitivity increase by HMGB1 in breast cancer. Acta Pharmacol Sin 2007, 28(12):1957-1967.

65. Rhodes DR, Yu J, Shanker K, Deshpande N, Varambally R, Ghosh D, Barrette T, Pandey A, Chinnaiyan AM: Large-scale meta-analysis of cancer microarray data identifies common transcriptional profiles of neoplastic transformation and progression. Proc Natl Acad Sci USA 2004, 101(25):9309-9314.

66. Wang W, Jiang $H$, Zhu H, Zhang $H$, Gong J, Zhang L, Ding Q: Overexpression of high mobility group box 1 and 2 is associated with the progression and angiogenesis of human bladder carcinoma. Oncol Lett 2013, 5(3):884-888.

67. Ouellet V, Le Page C, Guyot MC, Lussier C, Tonin PN, Provencher DM, MesMasson AM: SET complex in serous epithelial ovarian cancer. Int $J$ Cancer 2006, 119(9):2119-2126.

68. Yu M, Stott S, Toner M, Maheswaran S, Haber DA: Circulating tumor cells: approaches to isolation and characterization. J Cell Bio/ 2011, 192(3):373-382.

69. Bianchi ME, Manfredi AA: High-mobility group box 1 (HMGB1) protein at the crossroads between innate and adaptive immunity. Immunol Rev 2007, 220:35-46.

70. Raucci A, Palumbo R, Bianchi ME: HMGB1: a signal of necrosis. Autoimmunity 2007, 40(4):285-289.

71. Scaffidi P, Misteli T, Bianchi ME: Release of chromatin protein HMGB1 by necrotic cells triggers inflammation. Nature 2002, 418(6894):191-195.

72. Huang MY, Wang HM, Tok TS, Chang HJ, Chang MS, Cheng TL, Wang JY, Lin SR: EVI2B, ATP2A2, S100B, TM4SF3, and OLFM4 as potential prognostic markers for postoperative Taiwanese colorectal cancer patients. DNA Cell Biol 2012, 31(4):625-635.

73. Ballarino M, Jobert $L$, Dembele D, de la Grange $P$, Auboeuf $D$, Tora L: TAF15 is important for cellular proliferation and regulates the expression of a subset of cell cycle genes through miRNAs. Oncogene 2012, 32(39):4646-4655.
74. Lam LL, Emberly E, Fraser HB, Neumann SM, Chen E, Miller GE, Kobor MS Factors underlying variable DNA methylation in a human community cohort. Proc Natl Acad Sci USA 2012, 109(Suppl 2):17253-17260.

75. Byun HM, Nordio F, Coull BA, Tarantini L, Hou L, Bonzini M, Apostoli P, Bertazzi PA, Baccarelli A: Temporal stability of epigenetic markers: sequence characteristics and predictors of short-term DNA methylation variations. PLoS One 2012, 7(6):e39220.

76. Xu Z, Bolick SC, DeRoo LA, Weinberg CR, Sandler DP, Taylor JA: Epigenomewide association study of breast cancer using prospectively collected sister study samples. J Natl Cancer Inst 2013, 105(10):694-700.

doi:10.1186/1755-8794-7-8

Cite this article as: Koestler et al:: Integrative genomic analysis identifies epigenetic marks that mediate genetic risk for epithelial ovarian cancer. BMC Medical Genomics 2014 7:8.

\section{Submit your next manuscript to BioMed Central and take full advantage of:}

- Convenient online submission

- Thorough peer review

- No space constraints or color figure charges

- Immediate publication on acceptance

- Inclusion in PubMed, CAS, Scopus and Google Scholar

- Research which is freely available for redistribution 